\section{A clear suspect}

Approximately $0.05 \%$ of the population of the Western world suffers from systemic lupus erythematosus (SLE) - a complex autoimmune disease. Although several susceptibility loci for SLE have been identified, the nature of the genes and mutations that underlie this disease has remained unknown. Now, Prokunina et al. report in Nature Genetics an association between programmed cell death gene 1 (PDCD1) and SLE. Importantly, they also propose how a particular sequence variant of PDCD1 might contribute to the aetiology of this disease.

In a previous study of a Nordic population, the authors identified a susceptibility locus for SLE on chromosome 2. One gene in this region stood out as a potential candidate PDCD1. This is because PDCD1 encodes an immunoreceptor, also known as PD1, that belongs to the CD28 family and is known to regulate peripheral self-tolerance of $\mathrm{T}$ and B cells. Moreover, $P d c d 1^{-/-}$mice suffer from SLE-like symptoms.

By sequencing $P D C D 1$ in five healthy, unrelated individuals and in five patients with SLE from a Nordic population, the authors discovered seven single-nucleotide polymorphisms (SNPs) in this gene, three of which constitute a diseaseassociated haplotype that can account for all of the genetic linkage seen in the original population sample. These SNPs were then genotyped in five sets of families of different ethnic origin. The results were clear - only one SNP, which is found in an enhancer-like region in intron 4 of $P D C D 1$, was associated consistently with SLE. This region of intron 4 contains binding sites for transcription factors that are known to be involved in haematopoietic differentiation and in inflammation. In particular, the SNP disrupts a putative binding site for RUNX1, a member of the Runt-related family of transcription factors. Using an electrophoretic mobility-shift assay, the authors confirmed that RUNX1 binds this sequence and that binding is abolished by the sequence change that is associated with the SNP.

The authors propose that binding of RUNX1 to wild-type PDCD1 modulates its transcription and ensures its correct expression. As PDCD1 contains an immunoreceptor tyrosinebased inhibitory motif (ITIM), it might be involved in preserving selftolerance by inhibiting autoreactive cells. It remains to be confirmed whether, in the absence of RUNX1 binding, dysregulation of PDCD1 leads to loss of self-tolerance and to the chronic lymphocyte hyperactivity that is characteristic of SLE.

Magdalena Skipper, Associate Editor, Nature Reviews Genetics

(D) References and links ORIGINAL RESEARCH PAPER Prokunina, L. et al. A regulatory polymorphism in $P D C D 1$ is associated with susceptibility to systemic lupus erythematosus in humans. Nature Genet. 28 October 2002 (DOl: 10.1038/ng1020)

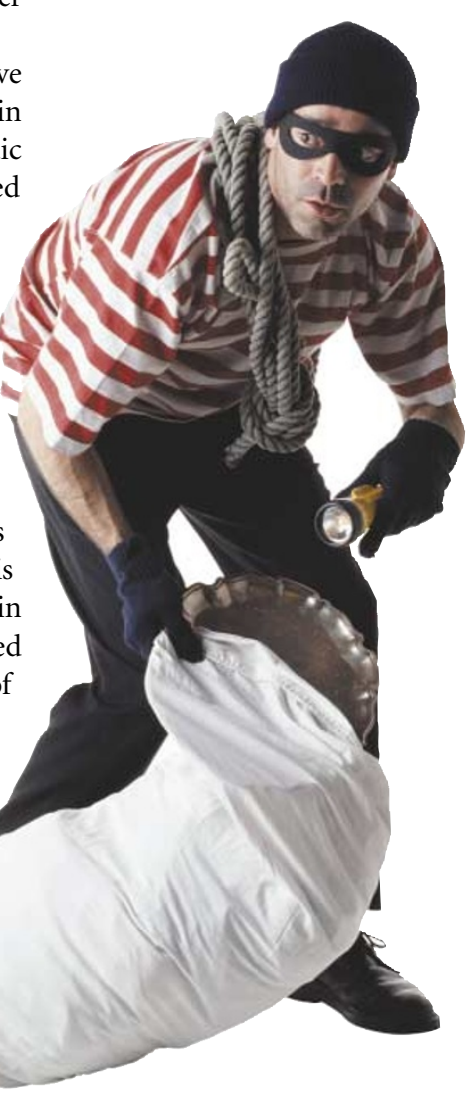

IN BRIEF

\section{LYMPHOID ARCHITECTURE}

Manipulation of lymphoid microenvironments in nonhuman primates by an inhibitor of the lymphotoxin pathway.

Gommerman, J. L. et al. J. Clin. Invest. 110, 1359-1369 (2002)

Continuous lymphotoxin (LT) signals are essential for the maintenance of lymphoid architecture and the network of follicular dendritic cells (FDCs). This reticular network has been implicated in the pathogenesis of HIV and prion diseases, as well as certain lymphomas and autoimmune diseases, so is inhibition of LT a potential therapy? Gommerman et al. show that in monkeys treated with human LT $\beta$-receptor-immunoglobulin fusion protein, the FDC networks disappeared within several days and affinity maturation of antibodies was impaired.

\section{APOPTOSIS}

\section{TRAIL/Apo-2 ligand induces primary plasma-cell} apoptosis.

Ursini-Siegel, J. et al. J. Immunol. 169, 5505-5513 (2002)

At the end of an immune response, most antibody-secreting cells (plasma cells) undergo apoptosis, but the mechanisms that regulate this process are not understood. This study indicates that the death receptor TRAIL might have a specific role in the elimination of plasma cells. Similar to resting and activated B cells, plasma cells were shown to express TRAIL, but only plasma cells were susceptible to ex vivo TRAIL-mediated killing.

\section{HIV}

In vivo dynamics of T-cell activation, proliferation and death in HIV-1 infection: why are $\mathrm{CD}^{+}$but not $\mathrm{CD}^{+}$ T cells depleted?

Ribeiro, R. M. et al. Proc. Natl Acad. Sci. USA 99, 15572-15577 (2002)

Deuterated glucose has been used to compare the dynamics of T-cell turnover between HIV-infected and -uninfected individuals. Here, Ribeiro et al. use a mathematical model, which takes into consideration the fact that only a fraction of $\mathrm{T}$ cells are proliferating at any given time, to analyse these data. This study provides insights into the differences between $\mathrm{CD} 4^{+}$and $\mathrm{CD} 8^{+}$ T-cell dynamics during HIV-1 infection.

\section{IMMUNOTHERAPY}

Gene therapy for Wiskott Aldrich syndrome: rescue of T-cell signaling and amelioration of colitis upon transplantation of retrovirally transduced hematopoietic stem cells in mice.

Klein, C. et al. Blood 14 November 2002 (DOI 10.1182/blood-2002-05-1423)

Wiskott-Aldrich syndrome (WAS), an X-linked primary immunodeficiency, is caused by mutations in the WASP gene. This study shows that retroviral transduction of WASP ${ }^{-/-}$ haematopoietic stem cells with WASP can rescue the T-cell signalling defect that is seen in the absence of the protein. These results are encouraging for possible gene-therapy trials for WAS. 